\title{
Traitements non chirurgicaux des sialolithiases : sialendoscopie et lithotripsie extra-corporelle (LEG)
}

\section{RÉSUMÉ}

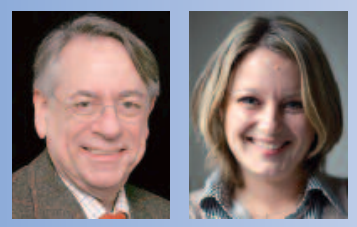

Philippe KATZ

Institut d'explorations fonctionnelles et d'endoscopie des glandes salivaires, 7, rue Théodore de Banville, 75017 Paris.

\section{Agnès GUERRE}

Institut d'explorations fonctionnelles et d'endoscopie des glandes salivaires.

Introduction. Les lithiases salivaires représentent une pathologie bénigne des glandes salivaires. Historiquement, seuls les traitements chirurgicaux étaient utilisés pour leurs prises en charge (ablation de la glande par voie transcutanée ou dissection des canaux excréteurs par voie endobuccale à la recherche des lithiases). Cette chirurgie n'est pas sans risque pour les structures avoisinantes (nerf facial ou nerf lingual essentiellement). Depuis vingt ans, le développement de l'endoscopie avec l'instrumentation endocanalaire et de la lithotripsie extracorporelle (LEC) a permis une nouvelle alternative à la chirurgie conventionnelle.

Matériel et méthodes. Nous avons pris en charge par ces techniques 3436 patients à I'Institut d'Explorations Fonctionnelles et d'Endoscopie des Glandes Salivaires (IEFGS) à Paris entre décembre 1988 et août 2010. Ils ont bénéficié d'un traitement par LEC dans 1571 cas et/ou par sialendoscopie dans 1865 cas. Ces deux techniques ont toujours été réalisées en ambulatoire, sans recours à l'anesthésie générale, tant chez les adultes que chez les enfants.

Résultats. L'endoscopie a permis d'extraire les calculs avec succès chez 96,4 \% des patients (1 402 sur 1455). Elle a aussi été utilisée pour le diagnostic voire le traitement d'autres pathologies comme les infections chroniques et les sténoses canalaires. La LEC a, quant à elle, permis de fragmenter $100 \%$ des lithiases en six séances en moyenne, 67,2 \% totalement et 32,8 \% partiellement. $92 \%$ des patients ont vu une disparition totale de leur symptomatologie rétentive, qu'il persiste ou non des fragments résiduels à l'échographie. Aucune complication majeure n'a été à déplorer.

- sialolithiase

- lithotripsie extra-corporelle

- endoscopie
AOS 2011;253:5-17

DOI: $10.1051 /$ aos/2011102

C AEOS / EDP Sciences 
Discussion. La réussite des procédures endoscopiques tient principalement en la sélection des bonnes indications (lithiase de taille inférieure à $4 \mathrm{~mm}$, canal de calibre suffisant et perméable, calcul non enclavé). La LEC a pu être effectuée sur toutes les lithiases avec une bonne efficacité. Les inconvénients principaux restent la longueur du traitement et la difficulté du repérage échographique qui rend la procédure opérateur-dépendant.

Conclusion. Le traitement des lithiases salivaires ne doit plus aujourd'hui faire appel au seul traitement chirurgical, mais doit prendre en compte les nouvelles techniques d'endoscopie et de LEC. Ces deux techniques sont particulièrement intéressantes et efficaces à condition d'en respecter les indications, affinées au fur et à mesure de leur développement et de notre expérience.

\section{Introduction}

\section{$>$}

Approximativement $1,3 \%$ de la population présente un ou plusieurs calculs salivaires. La localisation est submandibulaire dans $76 \%$ des cas et parotidienne dans $22 \%$ des cas. Les sialolithiases peuvent exister parfois dans la glande sublinguale ( $2 \%$ ). Dans $98 \%$ des cas, une seule glande salivaire est porteuse de lithiase.

L'âge d'apparition des symptômes varie de 3 à 85 ans, avec une moyenne de 44 ans [1].

La formation des calculs salivaires était classiquement décrite comme une infection chronique des glandes entraînant une diminution de la sécrétion salivaire avec formation dans un acinus d'une petite particule de mucus s'enrobant ensuite de phosphate de calcium ou d'autres sels minéraux pour donner une lithiase.

En fait, il apparaît qu'à l'interrogatoire de nos patients, plus d'un quart présentaient des antécédents familiaux avec au moins un parent ou un collatéral ayant eu une lithiase salivaire. Par ailleurs, dans notre série, 231 (14\%) patients de moins 10 ans avaient déjà une ou deux lithiases, sans antécédent patent d'infection salivaire. Dans ces conditions, il nous apparaît que les lithiases salivaires sont beaucoup plus d'origine génétique qu'infectieuse.

Sterenborg en 1990 [2] fit analyser 11 calculs pendant son étude sur la LEC et trouva que leur composition était de l'hydroxyapatite mélangée à d'autres sels minéraux. En 1996, Arzoz et al. [3] montrèrent entre autres que la composition des calculs était en majorité faite de phosphate de calcium (14 calculs) et pour un cas de cystine.

Historiquement, seuls les traitements chirurgicaux étaient utilisés dans le traitement des lithiases salivaires. Ils consistaient soit en l'ablation complète de la glande par voie transcutanée, soit en une dissection des canaux excréteurs par voie endobuccale à la recherche des lithiases. Cette chirurgie n'était pas sans risque pour les structures avoisinantes telles que le nerf facial pour la glande parotide ou le nerf lingual pour la glande submandibulaire. Le développement de la sialendoscopie et de la LEC a permis une alternative à la chirurgie conventionnelle. 


\section{Endoscopie}

\section{Introduction}

L'endoscopie des glandes salivaires a pu être réalisée grâce aux progrès de la technologie et à la fabrication de fibroscopes flexibles ultrafins de moins d'un millimètre de diamètre.

Nous avons réalisé la première endoscopie mondiale en décembre 1988. II s'agissait d'une endoscopie diagnostique au niveau d'une glande parotide, elle fut publiée en 1990 [4].

En 1991 et 1993 [5, 6], nous avons rapporté les premières séries d'endoscopies thérapeutiques permettant l'ablation de lithiases à l'aide d'une sonde à panier miniaturisée.

Nahlieli et al. [7-12] ont décrit à de multiples occasions depuis 1994 leur propre technique, tant chez des adultes que chez des enfants. L'évolution des endoscopes interventionnels est clairement visible tout au long de ces années. Dans leur protocole, les calculs étaient de préférence extraits par voie endoscopique à l'aide de forceps, de pinces à panier ou encore par succion. Si le calcul ne pouvait être enlevé par I'un de ces moyens, alors il était fragmenté in situ à l'aide de pinces à mors coupants. Les échecs (13\%) étaient liés soit à une sténose trop serrée du canal, soit à une trop grande angulation du canal excréteur ou encore à des localisations aberrantes du calcul.

Marchal et al. [13, 14] ont publié leur expérience de sialendoscopie depuis 2001. La procédure décrite commençait par l'utilisation d'un endoscope semi-rigide de petit calibre servant au diagnostic et au repérage de la lithiase, puis un endoscope plus large avec canal opérateur introduisant ainsi une instrumentation endocanalaire type sondes à panier. Ils purent ainsi faire l'ablation de lithiases allant jusqu'à 4 mm de diamètre avec un taux de réussite de $96 \%$. Pour les calculs de plus gros diamètre, l'endoscopie diagnostique était suivie d'une lithotripsie intracorporelle sous endoscopie à l'aide d'ondes de chocs électrohydrauliques ou de fragmentation par laser type Holmium, sous anesthésie générale, avec la nécessité de réaliser plusieurs interventions dans $45 \%$ des cas.

\section{Matériels et méthodes}

Dans notre série, 1865 patients qui présentaient une pathologie salivaire ont été traités par endoscopie salivaire à l'Institut d'Explorations Fonctionnelles et d'Endoscopie des Glandes Salivaires (IEFGS).

Dans 1455 cas (78 \%), la pathologie était lithiasique, $1178(81 \%)$ localisations submandibulaires et 277 (19\%) localisations parotidiennes. Les autres cas correspondaient à des infections chroniques (145, soit 7,8 \%), des sténoses (115, soit $6,2 \%$ ), des ganglions (47, soit $2,5 \%$ ), des tumeurs (37, soit $2 \%$ ) et des glandes normales (66, soit $3,5 \%)$.

En effet, à partir de décembre 1988, 66 glandes salivaires réputées normales après échographie et sialographie (34 glandes submandibulaires et 32 glandes parotides) ont été examinées à visée diagnostique afin d'obtenir une anatomie endoscopique.

Les 1865 patients se composaient 924 hommes $(49,5 \%)$ et 941 femmes (50,5 \%). L'âge moyen des patients était de 44,5 ans, allant de 4 à 85 ans.

Les ablations de calculs salivaires ont commencé en avril 1990, après la création d'une pince à panier miniaturisée de $0,8 \mathrm{~mm}$ de diamètre (fig. 1 et 2 a à $c$ ). 

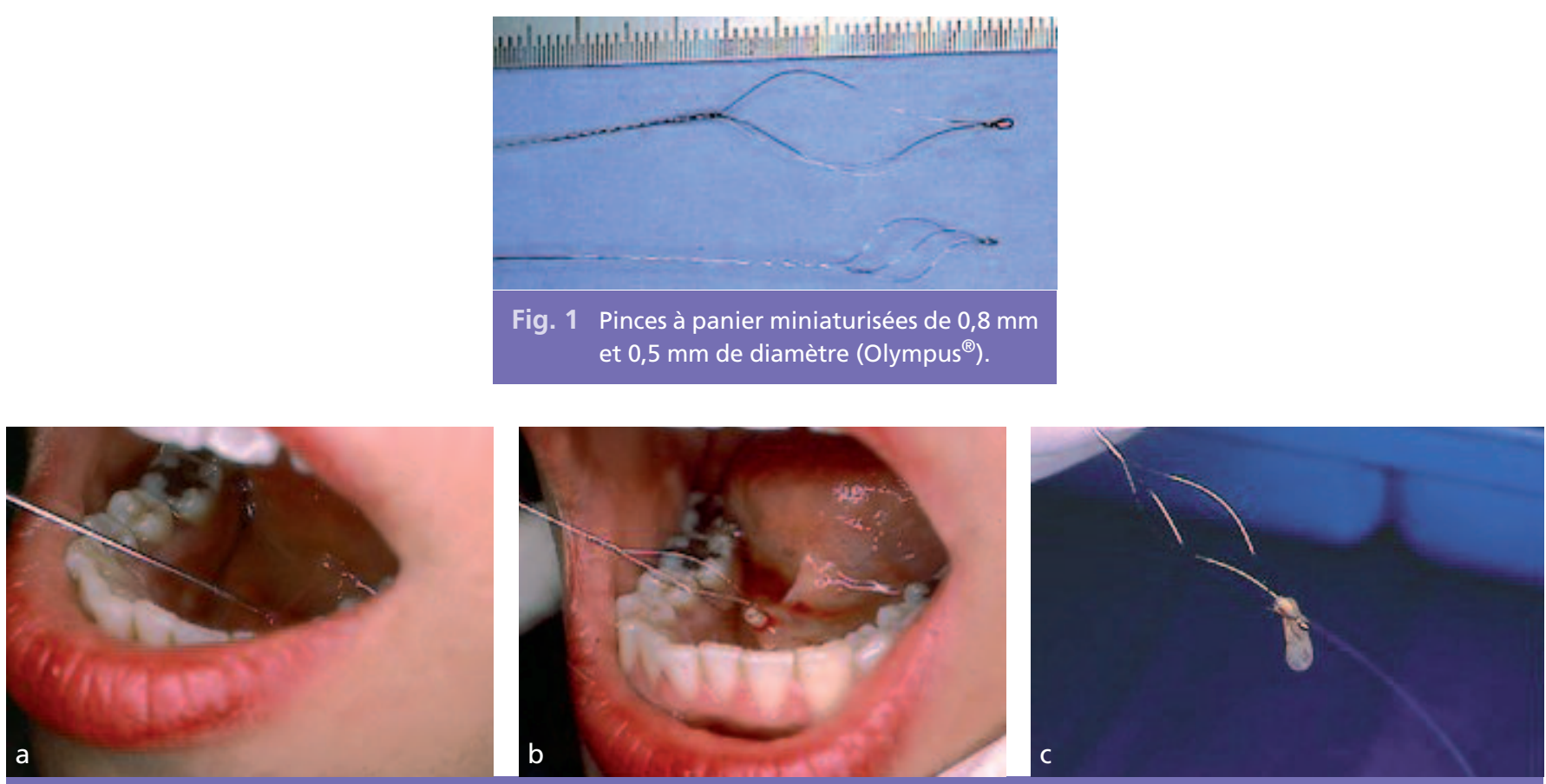

Fig. 2 a à c Mise en place de la sonde à panier dans le canal de Wharton et ablation du calcul.

Elles ont été réalisées à l'aide d'endoscopes ultra-fins souples de 0,4, 0,8 et 1,1 mm (fig. 3), puis avec un endoscope semi-rigide de $1,2 \mathrm{~mm}$ de diamètre utilisant une chemise avec canaux opérateurs (irrigation et instrumentation) (fig. 4).

Le protocole utilisé a toujours été le même depuis vingt ans. Nous pratiquons dans un premier temps le repérage de la lithiase. Pour cela, nous effectuons un cliché sans préparation (cliché endobuccal occlusal pour les glandes submandibulaires), ou mieux, une tomographie volumétrique scannographique numérisée. Une échographie des glandes salivaires est réalisée systématiquement. Elle a pour but de visualiser le parenchyme glandulaire et d'évaluer sa fonctionnalité (souffrance ou atrophie glandulaire). Elle permet aussi une localisation précise de la lithiase et de son retentissement sur les canaux excréteurs (fig. 5 a et b).
Nous pratiquons ensuite, après traitement d'une potentielle infection, une sialographie pour mesurer le diamètre du canal excréteur et s'assurer de l'absence de sténose canalaire ainsi que pour évaluer le retentissement de l'obstacle lithiasique sur les canaux excréteurs d'amont (fig. 6 a et b).

Si toutes les conditions sont réunies, c'est-à-dire un canal excréteur perméable et de calibre suffisant à l'introduction de l'endoscope, une lithiase mobile ou mobilisable et une glande salivaire non atrophique et fonctionnelle, nous envisageons la sialendoscopie.

L'introduction du fibroscope se fait après dilatation simple de l'ostium à l'aide de sondes calibrées atraumatiques. Aucune anesthésie n'est nécessaire pour l'introduction de l'endoscope et le repérage de la lithiase. Devant l'extrême finesse du matériel endoscopique, il n'existe pas de béquillage et le guidage est effectué par des 

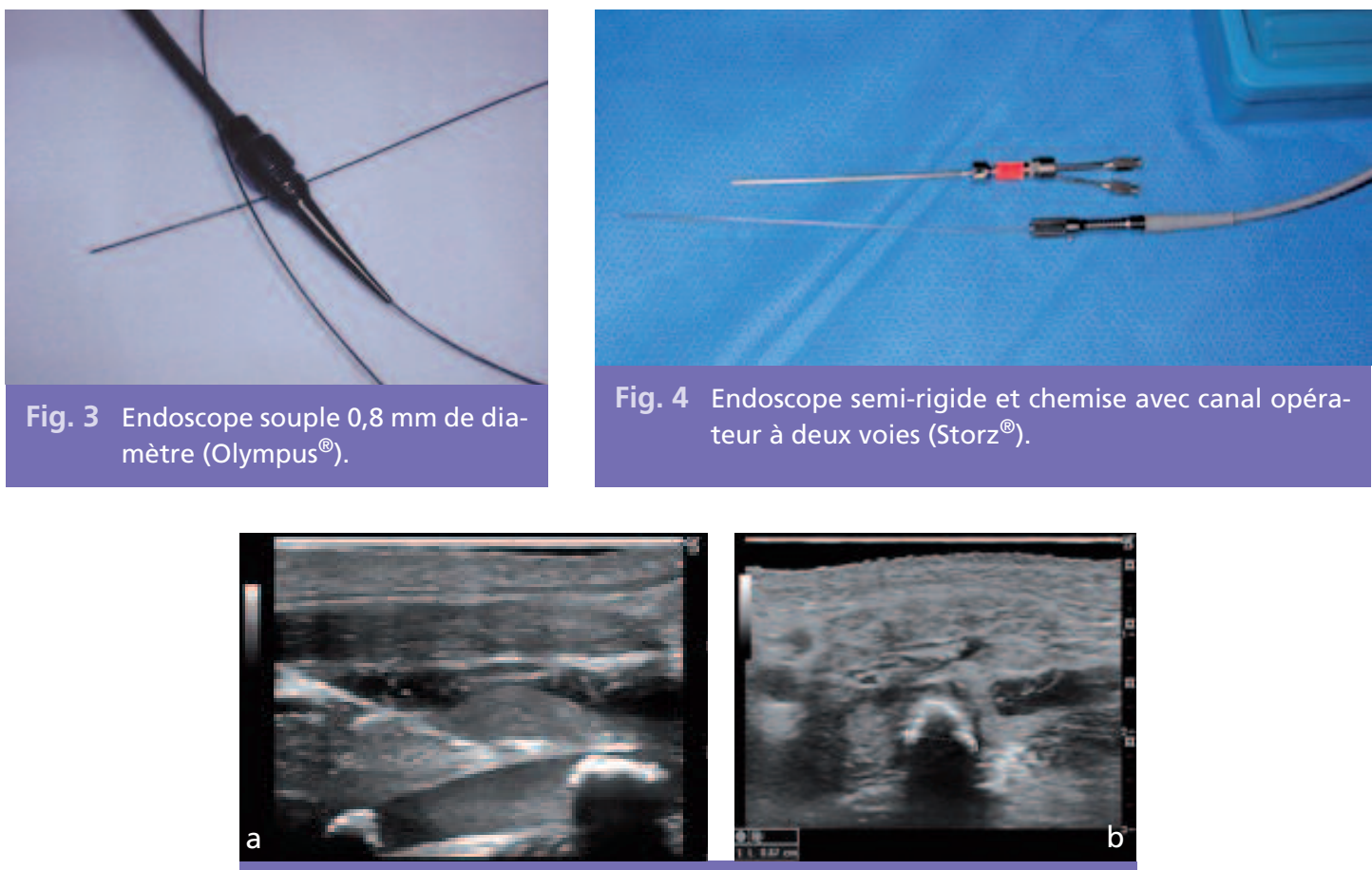

Fig. 5 a et b Images échographiques de lithiases submandibulaires (canalaires avec dilatation du canal [a] et du bassinet [8]).
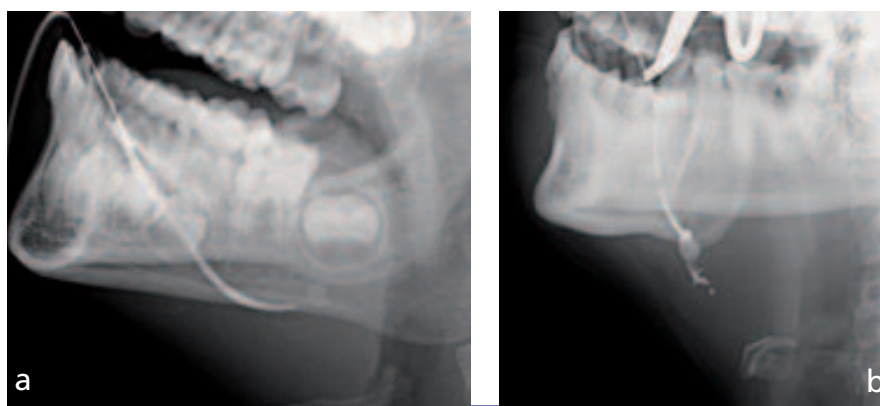

Fig. 6 a et b Sialographie de la glande submandibulaire avec lithiase dans le bassinet.

mouvements manuels par voie externe au contact de la peau ou de la muqueuse buccale. Une fois la lithiase repérée, l'introduction d'une sonde à panier miniaturisée sous contrôle endoscopique va permettre la préhension du calcul qui est ramené doucement vers l'ostium. Une papillotomie est presque toujours nécessaire à son extraction, cette dernière est faite sous anesthésie locale. Avant la papillotomie, la pro- duction salivaire physiologique sur un patient réveillé permet une bonne qualité de visualisation endocanalaire sans nécessité d'irrigation pendant la procédure. L'irrigation à la solution saline devient souvent utile après la papillotomie lors de la vérification endoscopique systématique de l'absence de lithiase résiduelle.

La procédure est toujours conduite en ambulatoire et sans arrêt de travail. 


\section{Résultats}

Depuis 1988, 1455 cas de sialolithiases ont été traités endoscopiquement, 1178 cas $(81 \%)$ au niveau de la glande submandibulaire et 277 cas (19 \%) au niveau de la glande parotide. Dans 233 cas (16\%), les lithiases étaient multiples, allant de 1 à 6 calculs. En effet, depuis 1995, initiation de la LEC, nous avons parfois combiné les deux techniques, et réalisé sous endoscopie des extractions de fragments lithiasiques obtenus par LEC (173 patients, 9,3\%).

Il a été possible de pratiquer 56 endoscopies chez des enfants âgés de 4 à 13 ans présentant des lithiases salivaires et d'en faire l'ablation (38 lithiases submandibulaires et 8 lithiases parotidiennes).

Au total, le nombre de patients pour lesquels I'extraction de la ou des lithiases a été faite avec succès était de 1402 sur 1455 , soit un taux de réussite de $96,4 \%$. Nous avons eu 53 échecs $(3,6 \%)$, dus à 19 glandes submandibulaires présentant des canaux de Wharton trop fins, à 13 glandes parotides dont il a été impossible de franchir le coude du muscle buccinateur et à 21 calculs enclavés dont la préhension n'a pas été possible.

L'endoscopie a aussi permis de mettre en évidence 102 (4,9\%) lithiases radio-transparentes, non visibles par les moyens radiologiques conventionnels. Ces lithiases ont toutes été extraites.

Aucun incident ou accident grave n'est survenu jusqu'à ce jour au cours d'une endoscopie, mais il faut savoir qu'une dilatation mal conduite peut entraîner une perforation du canal excréteur de la glande. Cet incident doit conduire à l'arrêt du geste.

L'endoscopie doit être pratiquée de la façon la plus douce et atraumatique possible pour éviter toute perforation canalaire ou glandulaire. Le franchissement d'un obstacle doit être réalisé après avoir examiné les parois du canal et apprécié le degré d'inflammation et l'élasticité des parois. Toute manœuvre brusque doit être prohibée.

Nous avons toutefois eu à déplorer au début de notre expérience un blocage d'une sonde panier dans une glande parotide qui a nécessité le recours à une procédure chirurgicale sous anesthésie générale.

\section{Discussion}

La phase de diagnostic est fondamentale. Elle doit permettre de mettre en évidence au niveau des glandes atteintes la meilleure méthode à choisir pour l'ablation de la lithiase. Idéalement, un canal perméable et de bon calibre, une lithiase mobile et de taille inférieure à 4 voire $5 \mathrm{~mm}$ et une glande fonctionnelle sont les meilleurs pronostics pour une endoscopie. Si ces conditions ne sont pas toutes réunies, I'endoscopie devra être pratiquée avec encore plus de précaution et de douceur, voire remise en question.

La réussite du geste dépend aussi de l'anatomie des canaux excréteurs, en effet un coude du buccinateur trop accentué sur le canal de Sténon ou un canal de Wharthon trop fin ne permettront pas la progression de l'endoscope.

Normalement aucune pathologie ne peut affecter la mise en place d'un endoscope ultra-fin dans une glande salivaire. La présence de pus franc dans le canal excréteur d'une glande peut altérer la vision, mais un lavage doux à l'aide de sérum physiologique rétablira les conditions normales.

La sialendoscopie est avant tout thérapeutique, mais elle peut aussi être diagnostique dans un 
premier temps en permettant la mise en évidence puis I'ablation de micro-lithiases radiotransparentes chez des patients souffrant de coliques salivaires et dont les examens radiologiques se sont révélés normaux.

L'efficacité de l'endoscopie est en général immédiate, entraînant après l'ablation du calcul une sédation des crises douloureuses. II peut cependant exister dans les premiers jours, un œdème papillaire ou une infection due au traumatisme canalaire, c'est pourquoi tous les patients bénéficient d'une antibiothérapie dans les suites du geste.

Un point important est à souligner, lorsqu'une glande salivaire a été mise en souffrance pendant une longue période par une lithiase, avec des infections chroniques, le fait de faire l'ablation de la lithiase entraîne une reprise de l'activité sécrétoire. Contrairement à ce qui était admis jusqu'à ce jour, les glandes salivaires ne sont que très rarement détruites complètement et leur activité peut reprendre lors de la levée de l'obstacle. Ceci a été parfaitement démontré sur des patients qui présentaient une atrophie glandulaire échographique et clinique avant l'ablation du calcul et qui ont récupéré une fonction salivaire sub-normale dans I'année

\section{Lithotripsie}

\section{Introduction}

La lithotripsie extracorporelle (LEC) des calculs salivaires est désormais possible grâce à la fabrication d'un matériel miniaturisé utilisant les ondes de chocs électromagnétiques de taille et de puissance adaptées aux sialolithiases et permettant leur fragmentation sans lésions glandulaires (fig. 7). suivant la levée de l'obstacle, avec une quasinormalisation des images échographiques.

Les limites de I'ablation d'un calcul par voie endoscopique, outre les conditions anatomiques dont nous avons parlé, sont principalement liées soit à la taille de la lithiase qui ne pourra pas être extraite par les voies naturelles si elle est trop grosse, soit à sa position enclavée dans le bassinet ou dans un canal primaire ou secondaire. Dans ces conditions, nous envisageons alors la seconde technique qui est la LEC.

\section{Conclusion}

Les procédures endoscopiques ont permis de traiter depuis leur initiation en 1988, 1865 patients, dont 1455 porteurs de lithiases uniques ou multiples. Elles ont d'abord été diagnostiques puis thérapeutiques, bénéficiant au fil des années d'améliorations technologiques, même si le protocole global est resté assez identique. La sialendoscopie a ainsi permis d'obtenir un traitement efficace chez $96,4 \%$ des patients porteurs de lithiases, les échecs étant dus à des indications difficiles où il aurait fallu discuter une autre technique.
Les premières expériences in vitro de fragmentation des calculs salivaires par lithotripteurs rénaux furent décrits en 1986 par Marmary [15] puis en 1989 par Brouns [16].

La fragmentation endocanalaire par différents lasers fut initiée et comparée par plusieurs auteurs [2, 3, 17-19] avec de bons résultats mais un risque important de plaies des canaux salivaires. La technique était parfois combinée 


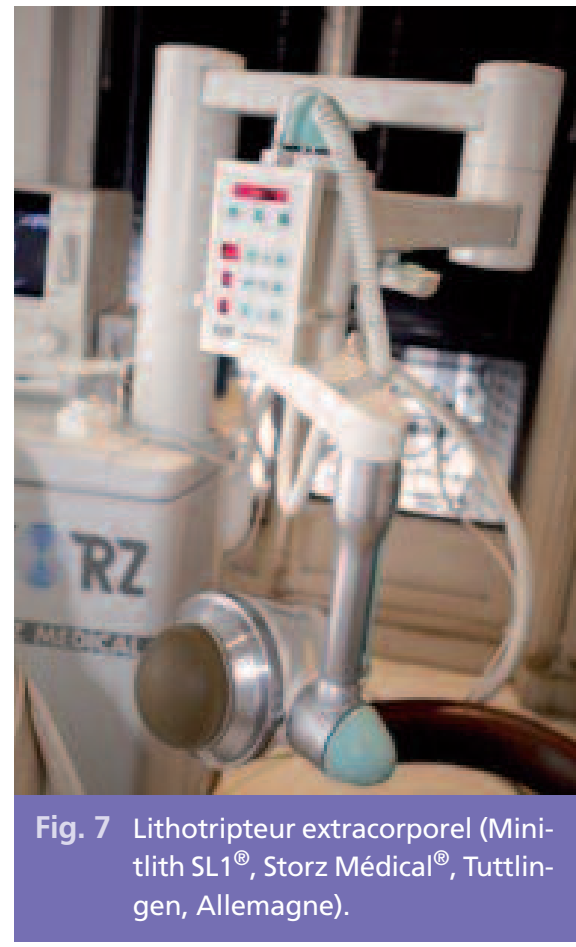

à l'endoscopie, sous anesthésie générale [13, 14].

En 1993, Konigsberger et al. [20] couplèrent avec succès et sans complications sur une série de 23 patients I'utilisation d'un endoscope rigide et d'un lithotripteur à ondes de choc électro-hydrauliques placé dans le canal à environ $1 \mathrm{~mm}$ devant le calcul.

En 1993 et 1996, Kater [21] puis Ottaviani [22] décrivirent leurs expériences de fragmentation extracorporelle par lithotripteur à ondes de choc électromagnétiques (Minilith $\mathrm{SL}-1^{\circledR} / \mathrm{K}$. Storz, Tuttlingen, Allemagne), qui est le matériel que nous utilisons depuis 1995. Dans la série d'Ottaviani [22], il s'agissait de 52 patients présentant des lithiases salivaires récusées pour l'endoscopie, dont $46 \%$ ont évacué complètement leur calcul, $31 \%$ ont conservé des fragments de moins de $2 \mathrm{~mm}$ et $23 \%$ des fragments supérieurs à $3 \mathrm{~mm}$ avec nécessité de réaliser une sialadénectomie dans un tiers des cas.

\section{Matériels et méthodes}

De juillet 1995 à août 2010, 1571 patients ont été traités par LEC à I'Institut d'Explorations Fonctionnelles et d'Endoscopie des Glandes Salivaires à Paris (IEFGS). Il s'agissait de 770 hommes (49\%) et 801 femmes (51\%), âgés de 6 à 85 ans, avec une moyenne de 45,5 ans.

Les lithiases submandibulaires, qu'elles soient uniques ou multiples, concernaient 1031 patients $(65,6 \%)$, avec une répartition égale des deux côtés.

Les lithiases parotidiennes étaient retrouvées chez 540 patients $(34,4 \%)$, dont 308 gauches et 232 droites. La taille des calculs variait de 2 à $34 \mathrm{~mm}$.

Le nombre de séances allait de 1 à 12 avec une moyenne de 6, espacées chacune de 3 à 24 mois, avec une durée de traitement allant de 3 mois à 6 ans.

Le protocole de prise en charge des patients a été le même depuis la mise en place de la technique.

Il consiste, comme pour l'endoscopie, en la réalisation première $d$ 'une imagerie radiologique standard ou d'une tomographie volumétrique scannographique numérisée, d'une échographie et d'une sialographie. Si l'indication de LEC est retenue, la première séance est réalisée après confirmation de l'absence de trouble de I'hémostase (dosage TP, TCA, Temps Saignement).

La séance de LEC est réalisée sans anesthésie sur un patient demi-assis. Elle débute par un repérage de la lithiase par sonde d'échographie de 7,5 MHtz, le centreur du lithotripteur est alors correctement placé et le tir commence. Le nombre d'impacts par séance va de 3000 à 6000 avec une fréquence de tir allant de 90 à $240 \mathrm{impacts} / \mathrm{min}$. L'onde de choc étant extrêmement focalisée $(2,5 \mathrm{~mm}$ sur $20 \mathrm{~mm})$ et son 
énergie adaptée aux calculs salivaires (5 à $30 \mathrm{mPa}$ ), les lésions sur les tissus avoisinants sont réduites.

Une antibiothérapie systématique est mise en place pour une durée de 10 jours après chaque séance. En cas de nécessité de plusieurs séances, un délai de 3 à 4 mois est respecté entre chaque fragmentation. Les contrôles échographiques sont systématiques et réguliers pour tous les patients.

Les fragments s'évacuent spontanément avec la salive, ou sont retirés par voie endobuccale avec sphinctérotomie de l'ostium du canal excréteur, à l'aide de pinces miniaturisées, avec une assistance endoscopique quand cela est nécessaire (fig. 8 à 10).

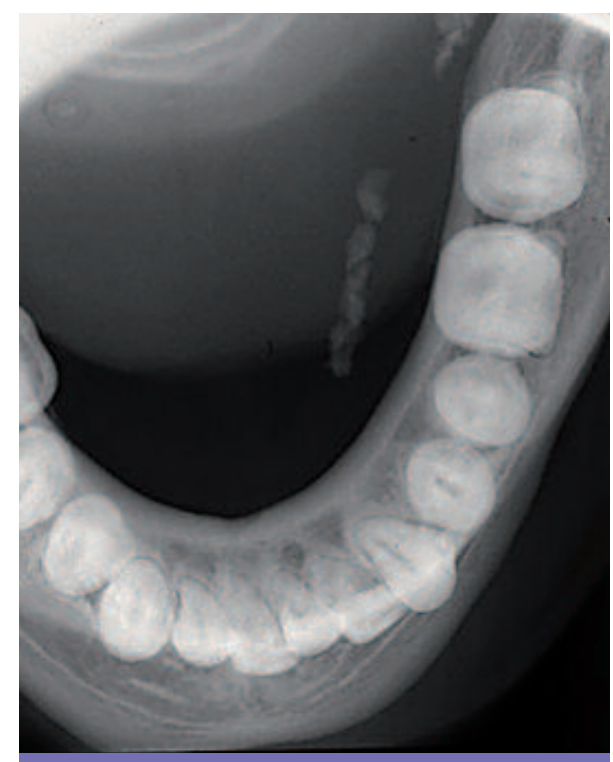

Fig. 8 Cliché occlusal montrant les fragments lithiasiques en cours d'évacuation dans le canal de Wharton après une LEC.

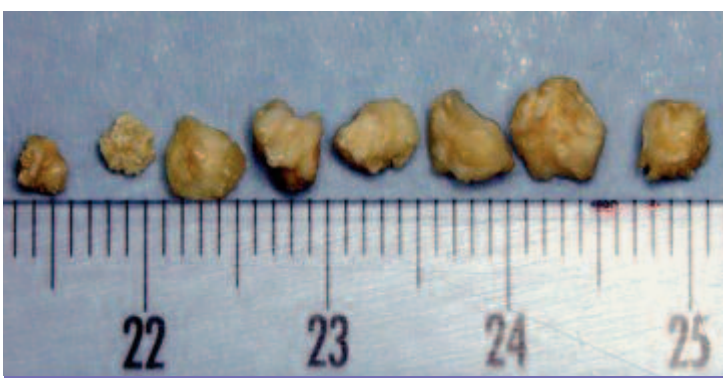

Fig. 9 Lithiase parotidienne fragmentée par lithotripsie.

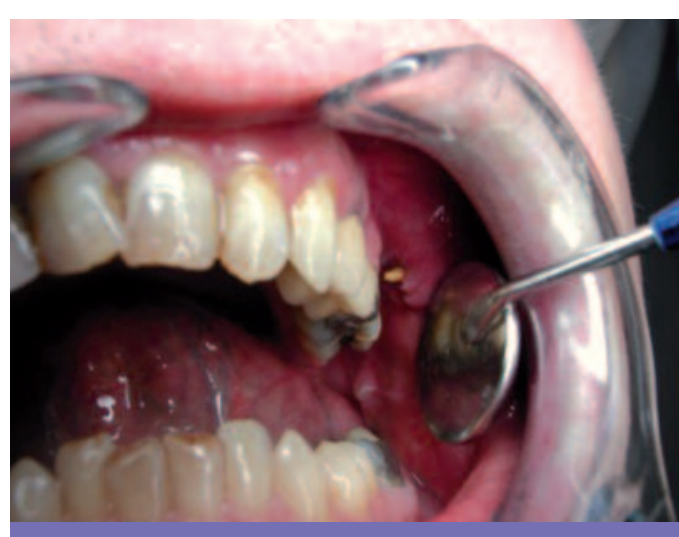

Fig. 10 Évacuation spontanée à l'ostium du sténon d'un fragment lithiasique parotidien dans les suites d'une LEC. 


\section{Résultats}

Toutes les lithiases ont été fragmentées dont $1056(67,2 \%)$ totalement détruites sans résidu et $515(32,8 \%)$ partiellement avec des fragments résiduels de 1 à $6 \mathrm{~mm}$.

Au niveau des glandes submandibulaires (1031 patients), les fragmentations totales étaient de $61 \%$ (629 patients) et les fragmentations partielles de $39 \%$ (402 patients).

Au niveau parotidien (540 patients), les fragmentations totales représentaient 427 cas sur $540(79 \%)$ et les fragmentations partielles 113 (21\%).

125 patients ont continué à présenter des coliques salivaires (8 \%) : 29 ont reçu des traitements antalgiques, antibiotiques et corticoïdes associé à des antispasmodiques au long cours et ont vu leurs symptômes diminuer $(1,8 \%)$; les 96 autres ont été perdus de vue $(6,1 \%)$.

Les effets indésirables temporairement observés au décours de la lithotripsie ont été les hémorragie canalaires spontanément résolutives (1 257 patients, ou $80 \%$ ), les pétéchies au point d'impact du tube (817 patients, ou $52 \%$ ) et les douleurs légères ( 597 , ou $38 \%$ ).

Dans les 15 jours qui suivent la lithotripsie, nous avons observé 628 cas (40\%) d'infections intraglandulaires, traitées par antibiotiques, corticoïdes et antispasmodiques.

II n'y a jamais eu de paralysie ou de paresthésie du nerf facial ou lingual, ni transitoire ni définitive, pas plus que de lésions dentaires liées aux ondes de choc.

\section{Discussion}

Il est désormais possible de fragmenter des calculs situés dans les glandes salivaires par voie extracorporelle grâce à la mise au point d'un appareil spécialisé le Minitlith SL1® ${ }^{\odot}$ (Storz Médi- $\mathrm{cal}^{\odot}$ ) destiné uniquement aux glandes salivaires.

La lithotripsie peut être effectuée sur tous les calculs salivaires quelle que soit leur taille et leur position. La taille de la lithiase n'a que peu d'importance, elle peut varier de $1 \mathrm{~mm}$ à $3 \mathrm{~cm}$, mais son repérage échographique doit être parfaitement effectué.

Il ne doit exister aucune infection intraglandulaire, au besoin un traitement anti-infectieux sera administré quelques jours avant la lithotripsie.

S'il apparaît que le canal excréteur de la glande est trop étroit (moins d'un millimètre), ne pouvant laisser les fragments s'évacuer, la LEC ne sera pas indiquée. C'est pourquoi la sialographie est l'examen radiologique fondamental avant toute thérapeutique. En effet, il est le seul capable de donner le calibre du canal excréteur et de s'assurer de son absence de sténose. La tomodensitométrie n'apporte que peu de renseignements sur le système canalaire glandulaire. La résonance magnétique nucléaire n'est d'aucune utilité.

Par ailleurs, il faut demander une exploration de la crase sanguine, car toute perturbation de l'hémostase peut contre-indiquer la LEC. En cas de traitement anticoagulant, son arrêt doit être discuté avec le médecin traitant ou le cardiologue. Concernant les effets indésirables, les infections glandulaires sont fréquentes (40\%), motivant une prescription antibiotique et corticoïde systématique après chaque séance.

Les autres effets indésirables transitoires (pétéchies au point d'impact du tube, douleurs légères s'associant à un gonflement de la glande, hémorragies canalaires spontanément résolutives) sont évalués comme mineurs par les patients eux-mêmes.

Il est important de signaler que, contrairement aux idées reçues, nous n'avons observé aucune 
lésion du nerf facial, transitoire ou définitive, aucune dysesthésie du nerf lingual, et aucune lésion dentaire à type descellement de prothèse ou fissure de l'émail.

Les fragmentations totales ont été plus fréquentes en parotidien (79 \%) qu'en submandibulaire (61\%), toutefois $92 \%$ de nos patients ne présentent plus de symptômes rétentifs, même s'il reste des fragments encore échographiquement visibles. Ceci s'explique par la reprise du flux salivaire normal à travers de petits fragments. En corollaire, chez les $8 \%$ de patients présentant encore des symptômes, 82 \% n'ont plus de fragments visibles à l'imagerie.

Sur ces patients symptomatiques, le traitement des crises à la demande a été mis en place avec une bonne tolérance. Le nombre de patients perdus de vue (96 soit 6,1 \%) s'explique par la longueur de la durée de notre étude. Certains ont certainement bénéficié d'un traitement radical par sialadénectomie dans une autre structure.

Les inconvénients de la LEC restent la longueur du traitement (nombre de séances important), la difficulté d'évacuation des fragments, la possibilité de laisser des fragments résiduels. La taille du calcul doit aussi faire discuter ce choix thérapeutique, en effet sur les lithiases centimétriques ou supra-centimétriques, on ne pourra souvent réaliser qu'une fragmentation partielle avec

\section{Conclusion générale}

La formidable évolution des nouvelles technologies non invasives a permis durant ces vingt dernières années de prendre en charge 3436 patients en leur évitant une chirurgie plus lourde. Le taux de succès d'ablation des lithiases submandibulaires et parotidiennes par endo- diminution du volume de la lithiase. Cependant, une fragmentation, même incomplète, permet le plus souvent une reprise du flux salivaire et une disparition de la symptomatologie rétentive. Enfin, nous rappellerons la fragilité extrême du parenchyme glandulaire salivaire et c'est la raison pour laquelle nous déconseillons toute tentative de lithotripsie sur des matériels non adaptés comme les tables de lithotripsie rénale, dont, ni le repérage du calcul, ni la puissance de fragmentation, ni la position du patient ne sont adaptés aux glandes salivaires et peuvent entraîner des lésions irréversibles.

\section{Conclusion}

Le diagnostic et la sélection des indications doivent être correctement analysés, afin d'utiliser la meilleure méthode. La fragmentation par LEC a pu être effectuée sur toutes les lithiases avec une bonne efficacité (92\%). Peu de contreindications sont décrites : infection aiguë, anomalie de la crase sanguine et canal excréteur infra-millimétrique ou sténosé ne permettant pas aux fragments de s'évacuer avec la salive. Les inconvénients restent la longueur du traitement et la difficulté du repérage échographique qui rend la procédure très opérateurdépendant.

scopie interventionnelle avec micro-instrumentation a été de $96,4 \%$ et celui de fragmentation par LEC de $92 \%$. Ces traitements sont donc une excellente alternative à la chirurgie conventionnelle à condition de les réserver aux bonnes indications. 


\section{Bibliographie}

1. Katz P, Fritsch $\mathrm{MH}$. Salivary stones: innovative techniques in diagnosis and treatment. Current Opin Otolaryngol Head Neck Surg 2003;11:173-8.

2. Sterenborg $\mathrm{H}$, Van den Akker $\mathrm{H}$, Van der Meulen C, et al. Laserlithotripsy of salivary stones: a comparison between the pulsed dye laser and HO-YSGG laser. Med J Sciences 1990;5:357-62.

3. Arzoz E, Santiago A, Esnal F, et al. Endoscopic intracorporeal lithotripsy for sialolithiasis. J Oral Maxillofac Surg 1996;54:847-50.

4. Katz, P.

Un nouveau mode d'exploration des glandes salivaires : la fibroscopie. Inf Dent 1990;8:785-6.

5. Katz P..

Endoscopie des glandes salivaires. Ann Radiologie (Paris) 1991;34(1):110-3.

6. Katz P.

Traitement endoscopique des lithiases salivaires. J ORL (Fr) 1993;42(1):33-6.

7. Nahlieli $O$, Neder A, Baruchin AM.

Salivary gland endoscopy: a new technique for diagnosis and treatment of sialolithiasis. J Oral Maxillofac Surg 1994;52:1240-2.

8. Nahlieli O, Baruchin AM. Sialoendoscope: three years experience as a diagnostic and treatment modality.
J Oral Maxillofac Surg 1997;55:912-8.

9. Nahlieli O, Baruchin AM. Endoscopic technique for the diagnosis and treatment of obstructive salivary gland diseases. J. Oral Maxillofac Surg 1999;57:1394-1401.

10. Nahlieli O, Eliav E, Hasson $\mathrm{O}$, et al. Pediatric Sialolithiasis. Oral Surg Oral Med Oral Path Oral Radiol Endodont 2000;90:709-12.

11. Nahlieli $O$, Baruchin AM. Long-term experience with endoscopic diagnosis and treatment of salivary gland inflammatory diseases. Laryngoscope 2000;988-93.

12. Nahlieli $O$, Shacham $R$, Yoffe B, et al.

Diagnosis and treatment of strictures and kinks in salivary gland ducts. J Oral Maxillofac Surg 2001;59:484-90.

13. Marchal $F$, Dulguerov $P$, Becker M, et al. Specificity of parotid sialendoscopy. Laryngoscope 2001;111:264-71.

14. Marchal $F$, Dulguerov $P$, Becker $\mathrm{M}$, et al. Submandibular diagnostic and interventional sialendoscopy: new procedure for ductal disorders. Ann Otol Rhinol Laryngol 2002;111:27-35.

15. Marmary $Y$.

A novel and non-invasive method for the removal of salivary gland stones. Int J Oral Maxillofac Surg 1986;15:585-7.

16. Brouns JJ, Hendrikx AJ, Bierkens AF. Removal of salivary stones with the aid of a lithotripter. J Craniomaxillofac Surg 1989;17:329-30.

17. Grundlach $P$, Scherer $H$, Hopf J, et al.

Die endoscopisch kontrollierte laserlithotripsie von speichelsteinen. HNO 1990;38:247-50.

18. Konigsberger R, Feyh J, Goetz A, et al. Die endoscopisch kontrollierte laserlithotripsie zur behandlung der sialolithiasis. Laryngo-Rhino-Otol 1990;69:322-3.

19. Iro H, Zenk J.

Laser lithotripsy of salivary duct stones. Adv Otorhinolaryngol 1995;49:148-52.

20. Konigsberger R, Feyh J, Goetz $A$, et al. Endoscopically-controlled electrohydraulic intracorporeal shock-wave lithotripsy (EISL) of salivary stones. J Otolaryngol 1993;22:12-3.

21. Kater W.

Die fortentwicklung des extrakorporalen stoss wellen lithotripsie von sperchelsteinen mit dem minilith.

(Meeting)

65 jahresversammlung des deutschen gesellschaff 
fur orl heilkumle kopf and halschirugie. Chemnitz, Germany, 5/1994.
22. Ottaviani F, Capaccio $P$, Campi $\mathrm{M}$, et al. Extracorporeal Electromagnetic shock-wave lithotripsy for salivary gland stones. Laryngoscope 1996;106:761-4.

\section{SUMMARY}

\section{Non-surgical Treatment of Sialolithiasis : Sialoendoscopy and Extracorporeal Shock Wave Lithotrypsy}

Philippe KATZ, Agnès GUERRE

\section{Keywords \\ - salivary gland calculi \\ - extracorporeal shockwave lithotripsy \\ - endoscopy}

Introduction: Up to recent times, treatment of salivary lithiasis has been based on surgery, whether open surgery through the neck or transoral dissection of the main salivary duct. This approach carries a certain morbidity, with a risk for the facial and lingual nerves. For the last 20 years, the development of endoscopic technology and extracorporeal shock wave lithotripsy (ECL) have improved possibilities for minimally invasive treatment of sialolithiasis, avoiding surgery.

Materials and Methods: 3436 patients were treated from December 1988 to August 2010 at the Institut d'Explorations Fonctionnelles et d'Endoscopie des Glandes Salivaires (I.E.F.G.S.) in Paris, France. ECL was employed for 1571 patients and sialendoscopy for 1865 patients, some patients receiving both treatments. Both treatements were administered on an outpatient basis, with only local anesthesia, for adults as well as for children.

Results: Sialoendoscopy was successful for lithiasis extraction in $96.4 \%$ of the cases $(1402 / 1455)$. Other pathologies were also treated at the same time with the same instrumentation, such as chronic infection and canal stenosis. ECL fragmented $100 \%$ of the lithiases over an average of 6 applications; $67.2 \%$ were completely destroyed and $32.8 \%$ partially fragmented. Symptoms completely resolved for $92 \%$ of the patients, whether or not residual fragments were visible on ultrasound. No major complication occurred.

Discussion: The success of sialendoscopy depends primarily on appropriate patient selection: lithiases smaller than $4 \mathrm{~mm}$, permeable salivary canals and lithiases that are not enclaved in the gland. ECL, on the other hand, was applicable to all types of lithiases. The major drawback of ECL is its operator-dependency, due to the need for precise ultrasonographic visualisation of the lithiasis, and the length of treatment.

Conclusion: Today, sialoendoscopy and ECL are major minimally invasive techniques for the treatment of sialolithiasis, and should be considered before considering a surgical approach. Both techniques are highly successful with appropriate equipment, patient selection and experience. 